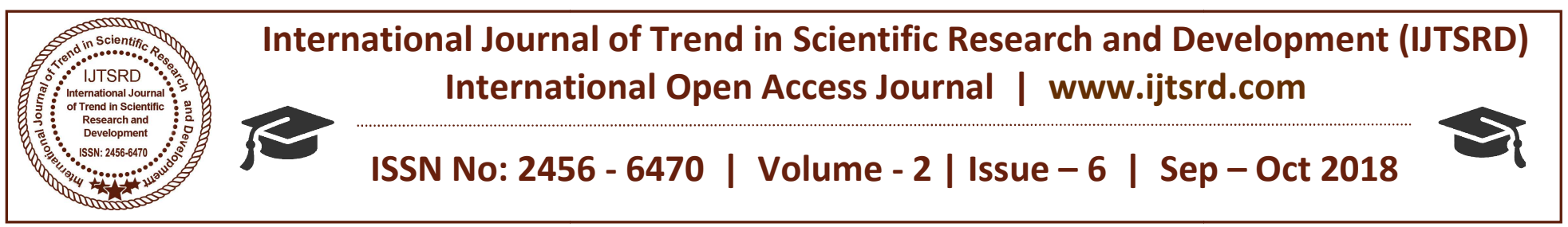

\title{
Heart Rate Variability Analysis of PC Interactive E-Learning Studies
}

\author{
Arjun Singh Vijoriya, Dr. Ranjan Maheshwari \\ Department of Electronics \\ Rajasthan Technical University, Kota, Rajasthan, India
}

\begin{abstract}
The objective of this particular research was the analysis of variations in the heart rate variability indicating the functionality of the autonomic nervous system as well as psychotic strain because of computer-based e-learning classroom study in the students. Comprehensive records were obtained from 25 students before and after the computer-based elearning classroom study for 2 hours having an average age of 21 years and in accordance with two comparable 8.46-minute HRV measurements of each student. The frequency domain and nonlinear analysis of HRV were conducted using the non-parametric FFT spectrum and Poincare plot analysis receptively on RR time series data extracted from ECG recordings. The HRV factors and details disclosed considerable variations in HRV before and after computer-based e-learning classroom study in students.
\end{abstract}

Keyword: Heart rate variability, E-learning, FFT spectrum, Poincare plot.

\section{INTRODUCTION}

The past two generations have experienced the identification of an important connection in between the autonomic nervous system (ANS) and cardiovascular fatality, consisting of unexpected cardiac fatalities [1-4]. Experimental confirmation for a connection with a tendency for venomous arrhythmias to symptoms of either greater sympathetic or minimal vagal function has inspired the evolution of quantitative indicators of the autonomic function. Heart rate variability (HRV) stands among the most encouraging such indicators. The seemingly simple derivation of this particular measurement has prominent its application. Among the various presented non-invasive methods for determining the autonomic status, HRV has emerged to be an ordinary technique to assess the sympathovagal equilibrium at sinoatrial level [6]. The sinus node is controlled by both parasympathetic (vagal) and sympathetic influences. Its well-accepted that situations such as for instance presuming an upright placement, mental anxiety, and exercising are linked to an enhancement of sympathetic tone. On the other hand, vagal tone is increased during the course of relaxing situations. In average people, both the sympathetic and the parasympathetic tone varies all over the day [7]. HRV indicator like the ratio of lowfrequency to high frequency power was used for describing sympathovagal harmony. In the lack of sympathetic and parasympathetic feedback to sinus node, sinus node shoots at the intrinsic rate or simply $\mathrm{R}-\mathrm{R}$ interval. Whenever vagal effect dominates, the heartrate is lower than intrinsic heart rate; whenever sympathetic effect dominate, heart rate is higher than intrinsic heart rate [4]. It was discovered that the HRV reduces along with age and with an individual, HRV is optimum during the course of sleep. It's also rate centered i.e. HRV is increased at reduced heart rates [8].

Several scientific studies have confirmed variations in HRV factors in disease situations. [9-11] The explanation of this is that the particular disease condition either causes, is caused by or is linked with irregularities of cardiac autonomic regulation. The diseased situation is connected with inherent mental pressure, not due to the pathological course of action of the disorder, but merely by stress and anxiety invoked in individuals as a consequence of consciousness of the disease or disorder. Anxiety induced fatigue is found to provoke the behavioral alerting consequence in human beings, that will be 
pertaining to boost in sympathetic activities and a drop in parasympathetic activities in the cardiovascular system. [12] This particular alternation in cardiac autonomic function alone could influence the HRV standards in patients. The time period of academic examination is a recognized model of mental pressure in scholars, overall performance in exams can determine their precious future potential. Various scientific studies state alterations in markers of anxiety in students throughout the period of examinations [13-15]. To examine the irregularities in HRV due to computer based e-learning that is caused by student's brain functional as well as phycho-emotional state after attending class, for this 25 students in the age group of 18 to 24 years old were analyzed before and after the regular computer based e-learning classes.

\section{Materials and Tools}

\section{A. Subjects}

Twenty-five students of single classroom age group of 18-24 (15 males and 10 female) volunteered to the part of this research were examined. A sample of time period 8.46 minute ECG data was recorded of each student before attending the e-learning class and after the continuous computer based e-learning class of basic computer knowledge of two hours. Each ECG recording of the particular student was taken on the same day in taking account of the timing and location. All the students were non-smoker, non-alcoholic, healthy and none of the student's family member was diabetic.

\section{B. Protocol}

All the recording was taken in the simple computer lab without any external interference like sound and scene. Subjects were stationary during the recording and sitting on the stools for 10 minutes. Subjects were instructed to do not change in their breathing unnecessary and to breath spontaneously in their regular rhythm. As a part of the study all the students were instructed to have only normal breakfast before attending the regular classes. Timing of the recording of all the students was in the morning between $7 \mathrm{am}$ to $11 \mathrm{am}$.

\section{Tools}

ECG data was recorded by the 3 lead ECG hardware having double stage amplification and interfaced with laptop by the use of Arduino Uno for real time data acquisition with the help of LabVIEW software installed in the laptop. LabVIEW recorded the real time ECG signal in laptop in dot tdms file format at the sampling frequency of $500 \mathrm{hz}$. This sampled data was processed by the ECG feature extractor in Biomedical Workbench of NI for signal processing and feature extraction and RR time interval was extracted in a .txt file which can be analyzed by the HRV analysis software. Heart rate variability analyzer in Biomedical workbench is used for HRV analysis which took data directly from the ECG feature extractor after the signal processing. HRV analyzer having the Poincare plot and FFT spectrum method of HRV analysis which generates values of HRV indices with different color coding [16]. As numerous commercial products nowadays furnish automated statistic of HRV measurement, the heart specialist has been presented that has seemingly easy tool both for research as well as clinical tests [5].

\section{Methods of analysis}

\section{A. Spectral Analysis}

There are multiple frequencies or frequency bands in the HRV analysis. Since 1960s there are various spectral analysis techniques have been evolved for the analysis of tachogram [17]. The important and basic information about the how power is dispersing in terms of frequencies; is generated by the power spectral density analysis of the RR time interval series by applying appropriate mathematical algorithms. There are to techniques are presented to calculated the PSD of the signal; first one is parametric method and second one is non-parametric method, these both techniques uses the different mathematical algorithms for computation. In this presented paper, FFT based non-parametric method is employed with the help of software as you can see a generated FFT spectrum of one subject in the figure (1). There are main three spectral components are recognized within the spectrum computed from RR time interval series recording after applying FFT based algorithm $[18,19,20,21,24]$ : The first spectral component is very low frequency (VLF) (0.003 to $0.04 \mathrm{~Hz})$, Second spectral component is low frequency (LF) (0.04 to $0.15 \mathrm{~Hz}$ ), and the third spectral component is high frequency (HF) $(0.15$ to $0.4 \mathrm{~Hz})$. The powers and fundamental frequency of all the frequency bands varies in accordance with the fluctuations in heart rhythms [21,22,23]. Dimension of power elements within VLF, LF and also HF bands is the absolute values of the power (ms2), although LF as well as HF can be calculated in normalized units (n.u.) [21,22]. 


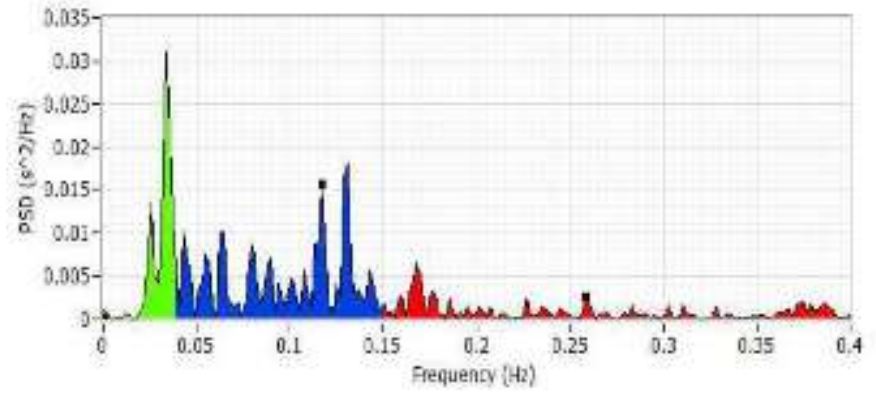

Fig. 1. HRV spectrum of sample data of one subject generated by HRV analyzer, where Green indicates the VLF spectrum, Blue indicates the LF and Red indicates the HF spectrum.

Based on the above RR-interval power spectrum Index of Centralization (IC) indicator is calculated which is associated with the brain's functional and phycho-emotional stress.

$\mathrm{IC}=((\mathrm{VLF}+\mathrm{LF})) / \mathrm{HF}$

where VLF, LF and HF are the Power spectral density in the respective frequency band. Increasing IC indicates the rise in heart rhythm $[37,38]$.

\section{B. Poincare Plot Analysis}

The Poincare plot as part of HRV is actually a scatter plot belonging to current R-R time interval plotted versus the prior R-R time interval. The Poincare plot can also abbreviate as Lorenz plot, scatter gram or Scatter plot and phase delay map or Return map. Poincare plot study is a simplified quantitative graphic approach in comparison to typical fast Fourier transform (FFT) exponent [27, 28]. The plot caters summary reports along with comprehensive beat-tobeat insight in regards to actions of the cardiovascular system (11). In this ellipse, points which are above the line are showing the intervals which are longer than preceding intervals and contrary points below the line showing intervals which are smaller the preceding interval.

Hence, the dispersal of point's vertical towards distinctive line of identity (width) exhibits the measure of interim HRV. The spots along with the distinctive line of identity (length) exhibit the longterm HRV [29]. Tulppo et al [30] equipped an ellipse in the form of the Poincare plot and also stated two typical descriptors of the entire plot, SD1 and then $\mathrm{SD} 2$, just for quantification of the entire geometry. Such standard descriptors portray the minor axis as well as the major axis on the ellipse correspondingly as displayed in Figure 2. The explanation of descriptors SD1 and SD2 with references to linear statistics, provided by Brennan et al [31] demonstrates that the typical descriptors assist the visual evaluation of the entire distribution. This exhibits an alluring pattern of the RR time interval data by portraying both the short as well as long term variations in the ECG signal $[30,31]$. SD1 reveals standard deviation of short term HRV in the data. The SD2 reveals standard deviation of progressive long term RR time intervals (major axis of ellipse) is determined with horizontal axis. SD1 signifies the instantaneous beatto-beat HRV and SD2 signifies the continual beat to beat HRV [32,33,34]. And the ratio of SD1/SD2 signifies randomness in heart rate variability (HRV) [35]. The location at which both axes contrast, represents total mean of RR time intervals. Several experts demonstrated that varying lags associated with Poincare plot. However, this will render better comprehension regarding the autonomic regulation of heart rate [27, 36]. After processing the recorded EGG data of students Poincare analysis was done by the use of HRV analyzer for each student record. A sample Poincare and its descriptors are show below for 8.46 minute ECG recording.

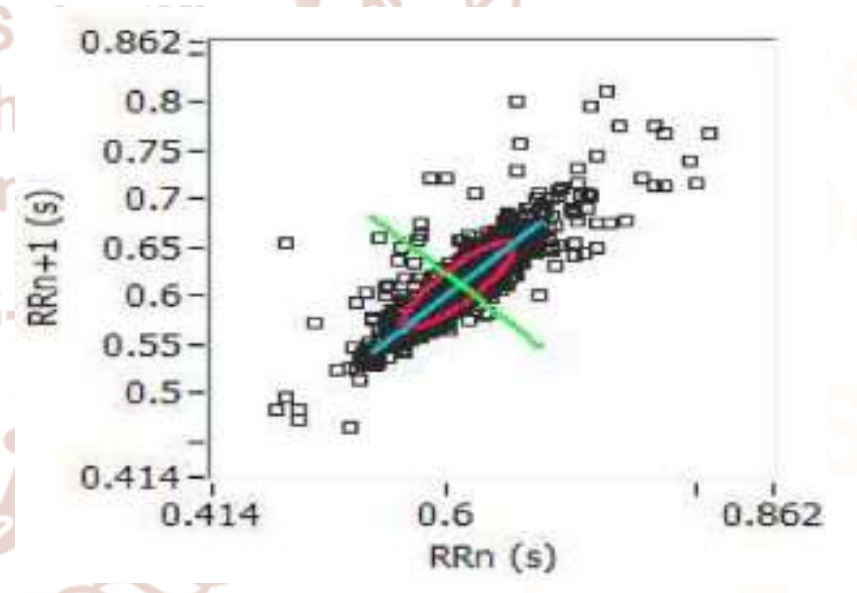

Fig. 2. Generated Poincare plot and values of SD1 $=16$ and $\mathrm{SD} 2=45$ for 8.46 minute $\mathrm{ECG}$ data by Biomedical Workbench's HRV analyzer, where green and bluish line depicts the SD1 and SD2 respectively.

\section{Results}

From the above tools and methods Non parametric FFT spectrum as frequency domain analysis is done for 25 students and power of frequency bands were calculated which are shown in the Table 1 . Where we can see the variations in the values of VLF, LF, HF and IC for before and after computer based e-learning classroom study. 
International Journal of Trend in Scientific Research and Development (IJTSRD) ISSN: 2456-6470

Table 1 Values of Power Spectrum Bands and Index of Centralization

\begin{tabular}{|c|c|c|c|c|c|c|c|c|}
\hline \multirow{2}{*}{ Suloject } & \multicolumn{3}{|c|}{ Before e-learning study } & \multicolumn{4}{|c|}{ After e-learning study } \\
\cline { 2 - 9 } & VLF Power & LF Power & HIF Power & IC1 & VLF Power & LF Power & HF Power & IC2 \\
\hline 1 & 250 & 730 & 825 & 1.187 & 480 & 1600 & 395 & 5.265 \\
\hline 2 & 110 & 280 & 284 & 1.373 & 140 & 440 & 225 & 2.577 \\
\hline 3 & 19 & 250 & 384 & 0.700 & 81 & 650 & 141 & 5.184 \\
\hline 4 & 72 & 420 & 93 & 5.290 & 390 & 900 & 171 & 7.543 \\
\hline 5 & 230 & 510 & 213 & 3.474 & 150 & 1100 & 260 & 4.807 \\
\hline 6 & 110 & 340 & 424 & 1.061 & 110 & 380 & 169 & 2.899 \\
\hline 7 & 44 & 360 & 71.2 & 5.674 & 570 & 790 & 147 & 9.251 \\
\hline 8 & 150 & 450 & 241 & 2.489 & 350 & 560 & 183 & 4.972 \\
\hline 9 & 390 & 770 & 227 & 5.110 & 52 & 200 & 33.8 & 7.455 \\
\hline 10 & 24 & 31 & 20.3 & 2.709 & 21 & 42 & 15 & 4.2 \\
\hline 11 & 47 & 740 & 290 & 2.713 & 22 & 330 & 93.9 & 3.748 \\
\hline 12 & 320 & 870 & 617 & 1.928 & 730 & 770 & 673 & 2.228 \\
\hline 13 & 130 & 1200 & 1010 & 1.316 & 130 & 670 & 316 & 2.531 \\
\hline 14 & 77 & 320 & 691 & 0.574 & 120 & 760 & 221 & 3.981 \\
\hline 15 & 23 & 160 & 168 & 1.089 & 15 & 43 & 14.7 & 3.945 \\
\hline 16 & 140 & 290 & 214 & 2.009 & 49 & 340 & 57.6 & 6.753 \\
\hline 17 & 130 & 450 & 881 & 0.658 & 35 & 140 & 114 & 1.535 \\
\hline 18 & 8.6 & 28 & 9.76 & 3.75 & 47 & 130 & 23.5 & 7.531 \\
\hline 19 & 20 & 620 & 199 & 3.216 & 100 & 350 & 79.8 & 5.639 \\
\hline 20 & 140 & 540 & 91.4 & 7.439 & 170 & 340 & 39.7 & 12.846 \\
\hline 21 & 42 & 250 & 63.9 & 4.569 & 66 & 240 & 47.5 & 6.442 \\
\hline 22 & 54 & 280 & 101 & 3.306 & 44 & 140 & 50.3 & 3.658 \\
\hline 23 & 22 & 160 & 84.6 & 2.151 & 12 & 150 & 62.6 & 2.587 \\
\hline 24 & 100 & 570 & 1140 & 0.587 & 150 & 460 & 709 & 0.860 \\
\hline 25 & 260 & 550 & 299 & 2.709 & 140 & 650 & 133 & 5.939 \\
\hline
\end{tabular}

A comparison graph can be plotted form above statistical data shown in Table 1 for values of IC for both before and after attending e-learning class of each student. The graph is shown in Fig. 3 which clearly showing the increment in the IC values of every student.

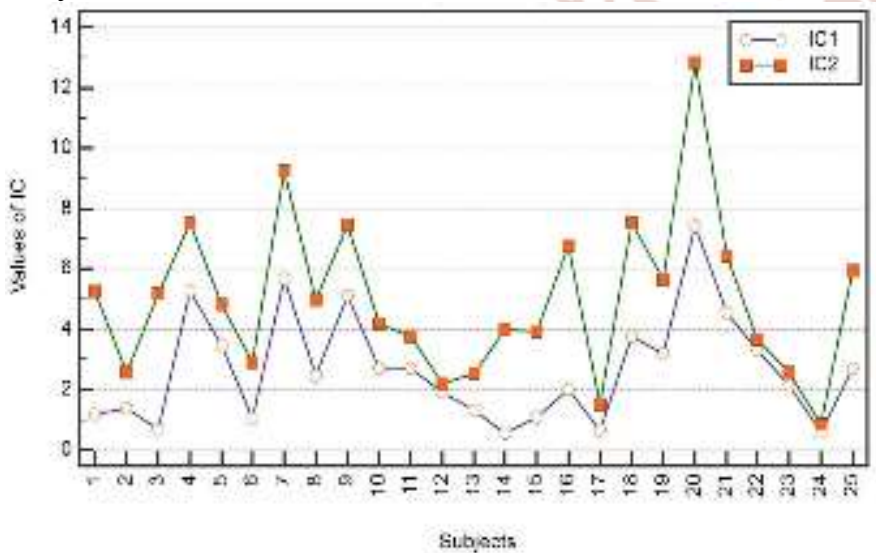

Fig. 3. Comparison graph for each student where IC1 is the value of IC before attending e-learning class and IC2, after attending e-learning class.
Using same tools Poincare plot analysis is done and values of $\mathrm{SD} 1, \mathrm{SD} 2$ and ratio the $\mathrm{SD} 1 / \mathrm{SD} 2$ are calculated for both time recording before attending the e-learning class and just after the e-learning class study without any other interference or activity in between and these Poincare plot descriptors for both are shown in Table 2. Which clearly shows the variation in the ratio SD1/SD2. We can easily observe the difference, there is the decrement in the magnitude of ratio SD1/SD2 after the computer-based e-learning classes. 
Table 2 Values of Descriptors Sd1 and Sd2 and their Ratio

\begin{tabular}{|c|c|c|c|c|c|c|}
\hline \multirow{2}{*}{ Sulject } & \multicolumn{3}{|c|}{ Before e-learning study } & \multicolumn{3}{|c|}{ After e-learning study } \\
\cline { 2 - 7 } & SD1 & SD2 & SD1/SD2 & SD1 & SD2 & SD1/SD2 \\
\hline 1 & 47 & 78 & 0.602 & 25 & 91 & 0.274 \\
\hline 2 & 26 & 51 & 0.509 & 17 & 44 & 0.386 \\
\hline 3 & 25 & 36 & 0.694 & 15 & 50 & 0.3 \\
\hline 4 & 13 & 41 & 0.317 & 15 & 52 & 0.241 \\
\hline 5 & 27 & 62 & 0.435 & 23 & 57 & 0.403 \\
\hline 6 & 33 & 61 & 0.540 & 21 & 47 & 0.446 \\
\hline 7 & 16 & 45 & 0.355 & 17 & 59 & 0.288 \\
\hline 8 & 20 & 50 & 0.4 & 19 & 67 & 0.283 \\
\hline 9 & 18 & 54 & 0.333 & 13 & 57 & 0.228 \\
\hline 10 & 17 & 39 & 0.435 & 7 & 20 & 0.35 \\
\hline 11 & 16 & 49 & 0.326 & 9.1 & 37 & 0.245 \\
\hline 12 & 36 & 74 & 0.486 & 37 & 85 & 0.435 \\
\hline 13 & 34 & 62 & 0.548 & 28 & 53 & 0.528 \\
\hline 14 & 28 & 61 & 0.459 & 20 & 55 & 0.363 \\
\hline 15 & 19 & 37 & 0.513 & 7.5 & 22 & 0.340 \\
\hline 16 & 25 & 53 & 0.471 & 12 & 44 & 0.272 \\
\hline 17 & 59 & 87 & 0.678 & 19 & 43 & 0.441 \\
\hline 18 & 7.8 & 21 & 0.371 & 5.7 & 25 & 0.228 \\
\hline 19 & 15 & 41 & 0.365 & 11 & 41 & 0.268 \\
\hline 20 & 18 & 52 & 0.346 & 8.1 & 43 & 0.188 \\
\hline 21 & 10 & 38 & 0.263 & 8.6 & 40 & 0.215 \\
\hline 22 & 12 & 42 & 0.285 & 9.2 & 33 & 0.278 \\
\hline 23 & 12 & 30 & 0.4 & 11 & 32 & 0.343 \\
\hline 24 & 33 & 62 & 0.403 & 25 & 65 & 0.384 \\
\hline 25 & 33 & 70 & 0.471 & 18 & 52 & 0.346 \\
\hline & & & & & & \\
\hline
\end{tabular}

Based on the data generated in Table 2, A comparison graph can be plotted for each student's SD1/SD2 ratio for both times before and after the attending elearning class, shown in figure (4) which depicts the increment in the ratio of SD1/SD2 for each student.

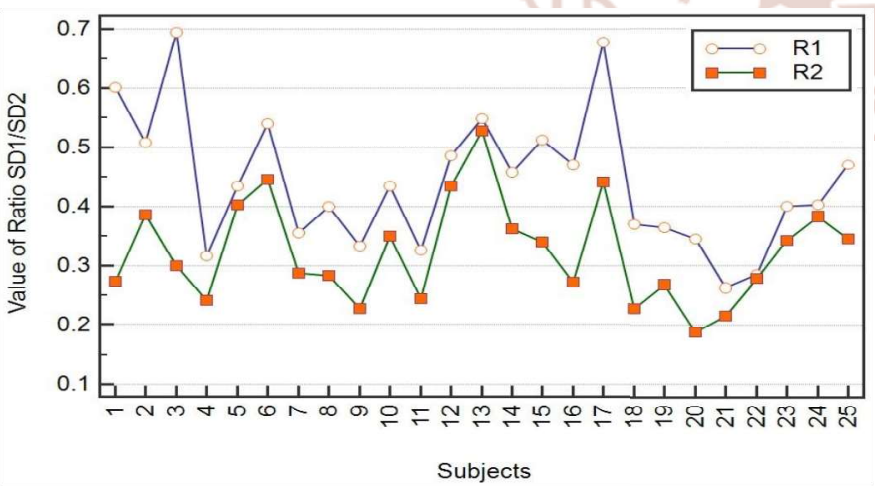

Fig. 4. Comparison graph of each student where R1 is value of ratio SD1/SD2 before attending e-learning class and R2, after attending the e-learning class.
V. Discussion

In the frequency domain analysis, the computation of index of centralization (IC) parameter by the use of VLF, LF and HF for both the times, before and the after PC interactive e-learning study of each student, demonstrates the brain's functional as well as phychoemotional state. Which means increment in the IC indicating the increment in the central control of the student's heart rhythm. [37, 38] From the Table 1, we can see that the value of IC of each student is incremented after the computer-based e-learning classroom study as compare to prior to attend the elearning class. Which indicates that computer-based e-learning causes the increment in the phychoemotional stress as well as brains functionally of each student that affects the heart rate variability.

In the Poincare plot analysis, descriptor SD1/SD2 ratio is used as a key parameter for analyzing the results. As previous studies show that the ratio $\mathrm{SD} 1 / \mathrm{SD} 2$ is lower for unhealthy persons which shows the more regular rhythm $[39,40]$. And in this 
presented study on 25 students, we noticed decrement in the ratio SD1/SD2 after the e-learning classroom study, from which can refer that with the computerbased e-learning having the adverse effect on the cardiovascular system.

\section{Conclusion}

Based on the research work done in this paper on this HRV analysis using two methods of analysis; both spectral analysis and Poincare plot analysis, this can be concluded that PC interactive e-learning studies causes the increment in the HRV which is induced by the brain's functional as well as phycho-emotional state of students. There could be numerous other factors can be involved that need further study on the same topic to identify other possible outcomes so that more precise and accurate result can be predicted based on the HRV analysis.

\section{ACKNOWLEDGMENT}

I would like to thanks to all the volunteer students who gave their valuable time and support for this particular research work.

\section{REFERENCES}

1. Lown B, Verrier R L. Neural activity and ventricular fibrillation. N Engl J Med 1976; 294: 1165-70.

2. Corr $\mathrm{P}$ B, Yamada $\mathrm{K}$ A, Witkowski $F / \mathrm{X}$. Mechanisms controlling cardiac autonomic function and their relation to arrhythmogenesis. In: Fozzard HA, Haber E, Jennings RB, Katz AN, Morgan HE, eds. The Heart and Cardiovascular System. New York: Raven Press, 1986: 13431403.

3. Schwartz P J, Priori S G. Sympathetic nervous system and cardiac arrhythmias. In: Zipes D P, Jalife J, eds. Cardiac Electrophysiology. From Cell to Bedside. Philadelphia: W. B. Saunders, 1990: 330-43.

4. Levy M N, Schwartz P J eds. Vagal control of the heart: Experimental basis and clinical implications. Armonk: Future, 1994.

5. Dreifus L S, Agarwal J B, Botvinick E H et al. (American College of Cardiology Cardiovascular Technology Assessment Committee). Heart rate variability for risk stratification of life-threatening arrhythmias. J Am CollCardiol 1993; 22: 948-50.

6. J. Sztajzel, "Heart rate variability: a noninvasive electrocardiographic method to measure the autonomic nervous system”, Swiss Med Wkly,134, 2004, pp. 514-522.

7. Jeffrey J. Goldberger, "Sympathovagal balance: how should we measure it?" Am. J. Physiol. 276 (Heart Circ. Physiol. 4), 1999, pp. H1273- H1280.

8. Awdah Al-Hazimi, Nabil Al-Ama, Ahmad Syiamic, ReemQosti, and Khidir Abdel-Galil, "Time domain analysis of heart rate variability in diabetic patients with and without autonomic neuropathy," Annals ofSaudi Medicine, 22 (5-6), 2002, pp. 400-402.

9. Mittal C M, Wig N, Mishra S, Deepak K K. Heart rate variability in human immunodeficiency viruspositive individuals. Int J Cardiol2004;94:1-6.

10. Dobrek L, Nowakowski M, Mazur M, Herman R M, Thor P J. Disturbances of the parasympathetic branch of the autonomic nervous system in patients with gastro esophageal reflux disease (GERD) estimated by short-term heart rate variability recordings. J PhysiolPharmacol2004; 55 (Suppl 2):77-90.

11. Chen J L, Chiu H W, Tseng Y J, Chu W C. Hyperthyroidism is characterized by both increased sympathetic and decreased vagal modulation of heart rate: Evidence from spectral analysis of heart rate variability. ClinEndocrinol (Oxf) 2006; 64:611-16.

12. Marshall J M. Cardiovascular changes associated with behavioural alerting. In: Jordan D, Marshall J (eds). Cardiovascular regulation. London: Portland Press; 1995:37-59.

13. Takatsuji K, Sugimoto Y, Ishizaki S, Ozaki Y, Matsuyama E, Yamaguchi Y. The effects of examination stress on salivary cortisol, immunoglobulin $\mathrm{A}$, and chromogranin $\mathrm{A}$ in nursing students. Biomed Res 2008; 29:221-4.

14. Johansson G G, Laakso M L, Peder M, Karonen SL. Examination stress decreases plasma level of luteinizing hormone in male students. Psychosom Med 1988; 50: 286-94.

15. Lucini D, Norbiato G, Clerici M, Pagani M. Hemodynamic and autonomic adjustments to real life stress conditions in humans. Hypertension 2002; 39:184-8.

16. Arjun Singh Vijoriya and Dr. Ranjan Maheshwari, ECG Signal Acquisition, Feature Extraction and HRV Analysis Using Biomedical Workbench, International Journal of Advanced Research in 
International Journal of Trend in Scientific Research and Development (IJTSRD) ISSN: 2456-6470

Engineering and Technology, 9(3), 2018, pp 8490.

17. Kay S M, Marple, S L. Spectrum analysis: A modern perspective Proc IEEE 1981; 69: 13801419.

18. Sayers B M. Analysis of heart rate variability. Ergonomics 1973; 16: 17-32.

19. Hirsh J A, Bishop B. Respiratory sinus arrhythmia in humans; how breathing pattern modulates heart rate. Am J Physiol 1981; 241: H620-9.

20. Akselrod S, Gordon D, Ubel F A, Shannon D C, Barger A C, Cohen R J. Power spectrum analysis of heart rate fluctuation: a quantitative probe of beat to beat cardiovascular control. Science 1981; 213: $220-2$.

21. Pagani M, Lombardi F, Guzzetti S et al. Power spectral analysis of heart rate and arterial pressure variabilities as a marker of sympatho-vagal interaction in man and conscious dog. Circ Res 1986; 59: 178-93.

22. Malliani A, Pagani M, Lombardi F, Cerutti S. Cardiovascular neural regulation explored in the frequency domain. Circulation 1991; 84: 1482-92.

23. Furlan R, Guzetti S, Crivellaro W et al. Continuous 24-hour assessment of the neural regulation of systemic arterial pressure and RR variabilities in ambulant subjects. Circulation 1990; 81: 537-47.

24. Kobayashi M, Musha T. 1/f fluctuation of heart beat period. IEEE Trans Biomed Eng 1982; 29: 456-7.

25. Saul J P, Albrecht P, Berger R D, Cohen R J. Analysis of long term heart rate variability: methods, $1 /$ f scaling and implications.

26. Computers in Cardiology 1987. IEEE Computer Society press, Washington 1988: 419-22.

27. Yamamoto Y, Hughson R L. Coarse-graining spectral analysis: new method for studying heart rate variability. J Appl Physiol 1991; 71: 1143-50.

28. Woo M A, Stevenson W G, Moser D K, Trelease RB, Harper RM. Patterns of beat-to-beat heart rate variability in advanced heart failure. AmHeart $\mathrm{J}$ 1992; 123: 704-710.

29. Woo M A, Stevenson W G, Moser D K, Middlekauff H R. Complex heart rate variability and serum norepinephrine levels in patients with advanced heart failure. J Am CollCardiol1994; 23: 565- 569 .

30. Brennan M, Palaniswami M, Kamen P. Poincare plot interpretation using a physiological model of HRV based on a network of oscillators. Am JPhysiol Heart CircPhysiol2002; 283: H1873H1886.

31. Tulppo M P, Makikallio $\mathrm{T} H$, Takala $\mathrm{T} E$, Seppanen T, Huikuri H V. Quantitative beat-tobeat analysis of heart rate dynamics during exercise. Am JPhysiol1996; 271: H244-H252.

32. Brennan M, Palaniswami M, Kamen P. Do existing measures of Poincare plot geometry reflect nonlinear features of heart rate variability? IEEE Trans Biomed Eng2001; 48: 1342-1347.

33. Brennan, M., Palaniswami, M., \&Kamen, P. (2001). Do existing measures of poincare plot geometry reflect nonlinear features of heart rate variabil- ity. IEEE Transactions on Biomedical Engineering, 48, 1342-1347. DOI: $10.1109 / 10.959330$.

34. Kitlas, A., Oczeretko, E., Kowalewski, M., \& Urban, M. (2004). Poincar'e plots in analysis of heart rate variability. PhysicaMedica, XX (Suppl. 1), $76-79$.

35. Piskorski, J., \&Guzik, P. (2007). Geometry of Poincar'e plot of RR intervals and its asymmetry in healthy adults. Physiological Measurement, 28, 287-300. DOI: 10.1088/0967-3334/28/3/005.

36. Biala, T., Godge, M., Schlindwein, F. S., \&Wailoo, M. (2010). Heart rate vari- ability using Poincar'e plots in 10 year old healthy and intrauterine growth restricted children with reference to maternal smoking habits during pregnancy. In Conference Proceeding: Computingin Cardiology, 26-29 September 2010 (pp. 971974). Belfast, Ireland.

37. Casolo G, Balli E, Taddei T, Amuhasi J, Gori C. Decreased spontaneous heart rate variability in congestive heart failure. Am J Cardiol1989; 64: 1162-1167

38. Uskov, V., Lyamin, A., Lisitsyna, L., Sekar, B.: Smart e-learning as a student-centered biotechnical system. In: Vincenti, G., Bucciero, A., Vaz de Carvalho, C. (eds.) eLEOT 2014. LNICST, vol. 138, pp. 167-175. Springer, Heidelberg (2014) 
39. Lisitsyna, L., Lyamin, A., Skshidlevsky, A.: Estimation of student functional state in learning management system by heart rate variability method. In: Neves-Silva, R., Tsihrintzis, G. A., Uskov, V., Howlett, R. J., Jain, L. C. (eds.) Smart Digital Futures 2014 - Frontiers in Artificial Intelligence and Applications, vol. 262, pp. 726731. IOS Press (2014)

40. Kitlas, A., Oczeretko, E., Kowalewski, M., \& Urban, M. (2004). Poincar'e plots inmanalysis of heart rate variability. PhysicaMedica, XX (Suppl. 1), 76-79.

41. Faust, O., Acharya, U. R., Molinari, F., Chattopadhyay, S., \& Tamura, T. (2012). Linear and non-linear analysis of cardiac health in diabetic subjects. Biomed-ical Signal Processing and Control, 7, 295-302. in DOI: 10.1016/j.bspc.2011.06.002. 